INVESTIGACIÓN / RESEARCH

Recibido: 22/01/2015-----Aceptado: 01/03/2014----- Publicado: 15/03/2015

\title{
TENDENCIAS PEDAGÓGICAS Y DIDÁCTICA: 4 VISIONES EN 4 TIEMPOS
}

Pedro Andrés Certad Villarroel ${ }^{\mathbf{1}}$ - Universidad Metropolitana, Caracas, Venezuela. pcertad@unimet.edu.v

\section{RESUMEN:}

El presente ensayo tiene por objetivo fundamental presentar las diversas visiones que tienen tanto de la didáctica como de la pedagogía: Jhon Dewey, B.F. Skinner, G. Lapassade y P. Freire, como representantes de una tendencia pedagógica (Escuela Nueva, Pedagogía de la Coacción, Pedagogía Institucional y Pedagogía Crítica, respectivamente). La aproximación a cada uno se realizó desde el análisis del discurso pedagógico de obras seleccionadas por su bibliometría resaltando las categorías: contexto histórico del autor; contenido ontológico, teleológico y axiológico; la didáctica: métodos, procedimientos, técnicas y recursos para la enseñanza y la evaluación, contenidos de la enseñanza, rol del docente, rol del alumno; la organización escolar: el ambiente de aprendizaje; y, por último, los conceptos fundamentales para comprender la teoría pedagógica del autor.

PALABRAS CLAVE: Pedagogía - Análisis del Discurso Pedagógico - Didáctica.

\section{TEACHING AND EDUCATIONAL TRENDS: 4 VISIONS IN 4 STROKES}

\section{ABSTRACT:}

This paper mainly aims to present the different views that the following authors have both in teaching and pedagogy: John Dewey, BF Skinner, G. Lapassade and P. Freire, as representatives of an educational trend (New School, Pedagogy of Coercion, Institutional Pedagogy and Critical Pedagogy, respectively). The

\footnotetext{
${ }^{1}$ Autor correspondiente:

Pedro Andrés Certad V: Facultad de Ciencias y Arte. Departamento de Ciencias de la Educación de la Universidad Metropolitana de Caracas. Venezuela.

Correo: pcertad@unimet.edu.ve
} 
approach to each ran from pedagogical discourse analysis of selected works by his bibliometrics highlighting categories: historical context of the author; ontological, teleological and axiological content; teaching: methods, procedures, techniques and resources for teaching and assessment, teaching content, teacher's role, role of the student; school organization: the learning environment; and finally, the fundamental concepts for understanding the pedagogical theory of the author.

KEYWORDS: Pedagogy - Pedagogic Discourse Analysis - Teaching

\section{INTRODUCCIÓN:}

Quizás, uno de los argumentos que mayormente he defendido se centra en que la educación actual, al menos a nivel universitario, presta mas atención a la didáctica que a la pedagogía; no solo desde la administraciones de los diseños curriculares sino también desde la esencia de la clase, desde el rol docente.

Es posible, que este separatismo se deba a la equivocada creencia que la pedagogía se orienta hacia los primeros años de vida del ser humano mientras que la didáctica es más amplia en su cobertura durante el hecho educativo y comprende mayor cantidad de aspectos formales que permean la planeación educativa, entonces cla didáctica es tangible y la pedagogía no?. Partiendo de esto, lo primero que me planteo es acercar al lector a evidencias de muestren que la pedagogía y la didáctica van de la mano, a partir de los resultados de un análisis del discurso pedagógico.

Simultáneamente, esa creencia que deambula en el camino híbrido de la psicología con la educación, ha pretendido dar parte preferente al aprendizaje como hecho cognitivo, construccionista o conductual, posiblemente buscando patrones, dejando a un lado lo complejo de la pedagogía y de la didáctica como su aplicación. Por ello, el segundo objetivo se pretende mostrar presenta la postura de psicólogos en el desarrollo de pensamientos pedagógicos, viendo que, a pesar de ser colindantes son diferenciables desde su aplicación; es decir, la pedagogía y la didáctica pueden apoyarse de teorías psicológicas pero éstas no son el todo del hecho educativo, son solo una parte del complejo multidisciplinar.

Se dan entonces visiones pedagógicas a través de cuatro representantes de distintas corrientes de pensamiento y tiempos: J. Dewey como parte de la Escuela Nueva, B. F. Skinner inmerso en la llamada Pedagogía de la Transmisión y la Coacción, G. Lapassade perteneciente a la Pedagogía Institucional y P. Freire como integrante de la Pedagogía Crítica. A partir de una obra de cada uno de estos 
maestros se mostrarán su ideas pedagógicas (Contenido ontológico, teleológico y axiológico), la didáctica aplicada (métodos, procedimientos, técnicas y recursos para la enseñanza y la evaluación, contenidos de la enseñanza) y, la relación pedagógica (rol del docente, rol del alumno. La organización escolar: el ambiente de aprendizaje) que se establece desde sus visiones dejando ver que el esmero en la pedagogía se mutualiza en la didáctica.

\section{LA ESCUELA NUEVA: JHON DEWEY}

John Dewey, filósofo cuya vida estuvo enmarcada entre tres grandes revoluciones originadas en la Ciencia: a) la Teoría Evolucionista propuesta por Darwin, b) la Teoría de la Relatividad de Einstein y, c) el inicio de la Era Atómica con los experimentos de Thompson, Rutherford y Niels Bohr. En todos los casos la idea del dinamismo en la vida y en el ambiente, el cambio continuo, la evolución y la adaptación se contraponen al pensamiento de las situaciones estáticas naturales como medio favorable para la observación científica que se venía desarrollando en el contexto de Dewey.

Durante su vida se caracterizó por ser un activista demócrata liberal y comunitarista, participando en grandes luchas políticas se inscribió en el movimiento Progresista mirando las posibilidades del futuro creyendo que la democracia estadounidense podía encontrar su realización en la reconstrucción de las instituciones sociales. Tanto él como los progresistas soñaban con una sociedad verdaderamente democrática y totalmente libre de la opresión la de la corrupción que provenían de las tradiciones feudales y autoritarias del viejo mundo. (Rockefeller, 1991)

Se hace presente en las ideas de Dewey el pensamiento de Hegel y así él mismo lo manifiesta: "la síntesis de Hegel de sujeto y objeto, materia y espíritu, lo divino y lo humano... representaron un inmenso alivio, una liberación". (Dewey, 1929 c.p. Dewey, 1938) Por otra parte, tal como se mencionó al principio, su apropiación de Darwin contribuyó a la redefinición del ser humano como un organismo adaptativo que transforma activamente su medio y se transforma a sí mismo, erigiendo el cambio como dimensión fundamental de la vida humano. Siendo anterior a Skinner define y separa la concepción dualista de estímulo y respuesta.

En la obra Experiencia y Educación, Dewey hace varias cosas a la vez. En primer lugar elabora un trabajo filosófico de reconstrucción de los conceptos centrales de la pedagogía. En segundo lugar, la valoración de la práctica pedagógica en función de los efectos que esta tenga en los alumnos y en por último la conceptualización de experiencia educativa como una crítica a la educación tradicional y al extremo progresista. 
En la obra de Dewey, tanto la Psicología como la Filosofía y la Pedagogía constituyen las tres vertientes que confluyen para formar la obra de Dewey. Desde la Psicología expone una suerte de manifiesto funcionalista sobre el acto reflejo y propone la separación del estímulo y la respuesta. La Filosofía la expone como un método para abordar los problemas de los hombres (Dewey, 1917 c.p. Dewey, 1938). En la Pedagogía, no cabe duda, fue un pensador avanzado a su tiempo, formando parte del movimiento de Escuela Nueva o Escuela Activa con un punto de complejidad, agudeza y radicalidad especial.

Para Dewey, la Pedagogía es el espacio donde se pone a prueba la filosofía del maestro como una forma reflexiva de valoración de estas prácticas a través de la observación y reflexión permanente por parte del maestro:

"Si estamos dispuestos a concebir la Educación como el proceso de formar disposiciones disposiciones fundamentales, tanto intelectuales como emocionales, hacia la naturaleza y hacia los demás, la filosofía puede ser definida como la Teoría General de la Educación. Si una filosofía va a dejar de ser simbólica, su valoración de la experiencia pasada y su programa de valores deben tener consecuencias sobre la conducta". (Dewey, 1918 c.p. Dewey, 1938)

No considera la Pedagogía como ciencia y prefiere concebirla como un arte que incluye a la ciencia dentro de sí misma, pues no cree que pueda transformarse en ciencia como la Ciencia Natural ya que las cuestiones humanas no son susceptibles a convertirse en reglas para la práctica pedagógica pues la misma experiencia del individuo es particular, no puede ser generalizada ni normada con otros individuos, esto forma una parte enriquecedora de su idea pedagógica pues debate el eficientismo y la generación de la medición que proponía Thorndike.

Con esta idea experiencial, Dewey pretende redefinir la pedagogía que hasta el momento se administraba en la escuela centrándose en cuatro ideas centrales de la práctica pedagógica: a) la experiencia educativa, b) el método de aprendizaje, c) el rol del maestro y d) el gobierno escolar.

Para Dewey la experiencia educativa viene a constituir la totalidad de las relaciones del alumno con el entorno. Esta experiencia no es necesariamente un evento de tipo cognitivo y en ésta el individuo actúa sobre el mundo y el mundo sobre el individuo, una relación activa y compleja que viene a determinar acciones educativas. Lo importante de esta experiencia es que no solo transforma al alumno en el momento, para Dewey existe una transformación en el pasado y en el futuro: "constituye una reconstrucción de la experiencia pasada y modifica la cualidad de las experiencias posteriores" (Dewey, 1938) 
Desde la didáctica, para Dewey las experiencias genuinamente educativas deben: incitar la curiosidad, fortalecer iniciativas y generar fuertes deseos para que en un futuro el alumno aborde los problemas que se le presenten ante cualquier otra experiencia y para valorar si esta experiencia es educativa el maestro debe fijarse en dimensiones de esa experiencia: la continuidad y el carácter interactivo: "Yo considero que la unidad fundamental de la nueva pedagogía se encuentra en la idea de que existe una íntima y necesaria relación entre los procesos de la experiencia real y la educación".

Las materias de enseñanza son para Dewey conjuntos de información y destrezas que han sido elaboradas en el pasado y la misión de la escuela es transmitirlos a las nuevas generaciones. Los libros de texto son los principales representantes del saber y la ciencia del pasado y el maestro son los órganos mediante los cuales el alumno se pone en una relación efectiva con las materias.

El propósito con que se diseñan las actividades inteligentes parte de la experiencia que ya tienen los alumnos y tiene en vista desde un principio su fin, supone una previsión de cada una de las consecuencias que nacerán en su desarrollo. La previsión de estas consecuencias supone para Dewey la inteligencia del individuo y parte de la observación de éste de las condiciones en las que se produce el fenómeno, posteriormente, la significación de lo observado, lo que trae a él la percepción de las consecuencias que resultarán cuando se actúe sobre lo que se observa conduciéndolo a obtener algún tipo de juicio que ponga en relación lo que se observa y lo que se recuerda para ver lo que significan. (Dewey, ob. Cit.)

En la relación pedagógica, la Escuela Nueva viene a ser la corriente y el ambiente en el cual la fuente primaria del control social reside en la misma naturaleza del trabajo realizado como una empresa social en la cual todos los individuos tienen la misma oportunidad de contribuir y respecto a la cual todos sienten responsabilidad. Se requiere un pensamiento y una planeación previa de las actividades y el educador es el responsable del conocimiento de los alumnos así como del conocimiento de las materias que se facilitarán, la selección de las actividades y la organización para que se produzca una igualdad en las oportunidades de participación.

El maestro debe seleccionar los conocimientos provenientes de las materias de estudio que sean de utilidad para el abordaje de los problemas surgidos de la experiencia de los alumnos y que a su vez estimulen nuevas formas de indagación. Es necesario reconocer que la pedagogía propuesta por Dewey revela un efecto liberador sobre la relación del maestro con el conocimiento. El maestro deja de ser concebido como un "administrador de la enseñanza" y de unas materias de estudio diseñadas para pasar a ser artista, académico, experimentador y creador de las condiciones necesarias para que una experiencia sea educativa garantizando la continuidad de ésta tanto con el pasado como con el futuro. Es necesario que esté 
atento a la dirección que toma la experiencia sin suprimir a los alumnos y sin que se transformen los propósitos para los cuales fueron ideadas las experiencias, también debe estar atento a las actitudes y hábitos que surjan durante la situación educativa conduciéndolos a un desarrollo continuado del alumno.

Específicamente en cuanto a la experiencia y la educación:

"Al maestro que une la educación con la experiencia presente incumbe una misión más seria y más difícil. Ha de conocer las potencialidades para dirigir a los alumnos a nuevos campos que se relacionan con experiencias ya vividas y ha de usar este conocimiento como criterio para seleccionar y disponer las condiciones que influyen en su experiencia presente" (Dewey, ob.cit.)

El alumno debe reflexionar sobre el proceso en su conjunto a fin de encontrar su sentido, entendido este como el "...capital acumulado para tratar inteligentemente ulteriores experiencias." (Dewey, Ob. cit.) El papel del alumno es activo y dinámico.

La propuesta pedagógica de Dewey fue evidentemente adelantada a su época, y aun hoy sigue siendo de difícil aplicación pues supone para muchos docentes una pérdida del control de la clase y un otorgamiento de facultades al alumnos que quizás ni este mismo pueda manejar. Se hace evidente que la idea del Conductismo ha calado fuertemente en el pensamiento del maestro. En otros casos la Escuela Nueva supone improvisación y rompe el paradigma de la clase preparada, planificada de la que no escapa ningún detalle, la clase del docente y no para el alumno.

\section{LA PEDAGOGÍA DE LA TRANSMISIÓN Y LA COACCIÓN: B. F. SKINNER}

Considerado como el segundo personaje, después de Freud, con más influencia en la psicología del siglo XX, y luego de las investigaciones de Thorndike y su principio de Ley de Efecto, con un particular humor, Burrhus Frederic Skinner (1904-1990) desarrolla un modelo formal de aprendizaje producido por la asociación entre una respuesta y su consecuencia, a lo que llamó Condicionamiento Operante.

Movido inicialmente en la psicología por la filosofía, Skinner lee diversas obras de Proust (literato francés influido por el impresionismo) lo que lo lleva a la autoobservación y posiblemente ha de haberle otorgado cierta inclinación hacia la Escuela de la Gestalt, pero tiempo más tarde, sus lecturas de la obras: Fisiología del Cerebro y Psicología Comparada (Loeb, 1900), Los Reflejos Condicionados (Pavlov, 1927), y Filosofía (Russel, 1925) lo conducen de la mano a simpatizar con 
el conductismo propuesto por Watson a través de la obra El Cuidado Psicológico de los Bebes y de los Niños. (Watson, 1928)

Para 1939, con la Segunda Guerra Mundial, se le presenta a Skinner la oportunidad de aplicar su idea del condicionamiento operante a los militares norteamericanos con un proyecto llamado Proyect Pigeon cuya intención fue la de mejorar los sistemas de armamento para lo que propone entrenar palomas para que guiasen los misiles, una idea un tanto descabellada pero para Skinner posible. También propone, a raíz de su experiencia como padre, la caja de Skinner en la publicación Baby in a Box, invento muy alabado pero también muy criticado quizás porque muchos científicos no se preocuparon por ir más allá de la simple visión directa del artefacto.

Hacia 1968 Skinner inicia la escritura de un nuevo libro "Más allá de la Libertad y la Dignidad" (Skinner, 1971, c.p. Bowen, 1986) defendiendo la postura de que el ser humano no puede ser libre: "Una persona nunca llega a ser autosuficiente de verdad" (Bowen, ob cit; p. 290), ya que la libertad es un concepto controlado por las contingencias ambientales introduciendo el hábil manejo de los conceptos de cultura y responsabilidad, las interacciones sociales o influencia social es importante para Skinner, lo que pone de manifiesto alguna traza de influencia ideológica proveniente de las ideas de Rousseau y Dewey acerca de dichas actividades sociales. Esta obra fue un completo bestseller en Estados Unidos ya que emerge en un momento controversial: la Guerra de Vietnam, los derechos de los negros, las protestas de género, entre otras, todas apuntando al incremento de la libertad y dignidad del ser humano.

Entre 1974 y 1984 editó su autobiografía en tres volúmenes: Particulars of my life" (1974), "The shaping of a behaviorist" (1979) y "A matter of consequences" (1984). También en 1974 publicó "Sobre el Conductismo", un libro cuyo objeto fue exclusivamente resolver de forma sencilla algunos de los principales errores y malentendidos que se habían ido desarrollando sobre el análisis de la conducta. También, llevando a cabo una recolección de artículos y trabajos, publicó tres libros: "Reflections on Behaviorism and Society" (1978), "Upon Further Reflection" (1987), "Recent issues in the Analysis of behavior" (1989).

Con el diseño y creación de la máquina de enseñar se deja ver que la Educación para Skinner tiene un componente tecnológico interesante, de hecho considera a la Educación como esa tecnología responsable de la construcción de la sociedad ideal y que debe reformularse en sus prácticas académicas, tal como lo refiere Bowen (1986):

Si se parte de las fascinantes perspectivas de una ciencia del aprendizaje en continuo avance, constituye una gran conmoción volcarse a esa rama de la tecnología que más directamente toca con el proceso de aprendizaje; la 
educación. (p. 278)... La educación es quizá la rama más importante de la tecnología científica y afecta profundamente las vidas de todos nosotros. ( $p$. 281) ... las técnicas que van apareciendo del estudio experimental del aprendizaje no están diseñadas para "desarrollar la mente" o para fomentar algún "entendimiento" vago ... están ideadas para establecer aquellas conductas precisas que toman como prueba de dichos estados y procesos mentales. (p. 284)

El aprendizaje es el proceso que de alguna manera modifica la vida del ser humano, la educación es la técnica o el conjunto de técnicas que será responsable de ayudar al cambio o fijación de una conducta. Es entonces cuando un estímulo es propiciado y captado por el aprendiz el cual va a producir una respuesta a dicho estímulo según el conocido esquema Estímulo - O - Respuesta (E-O-R), lo que pareciera no quedar especificado es la "caja negra", ese proceso, el qué pasa entre el estímulo y la respuesta en el individuo. Es posible que en esa caja (O) quepa lo que corresponde a la didáctica.

En cuanto al propósito de la educación para Skinner, se enmarca en la modificación de las conductas del ser humano y su finalidad se centra en el comportamiento esperado por parte de este ser humano que de alguna forma contribuya a la conformación de esa sociedad ideal. En consecuencia, los valores a desarrollar esta educación propuesta por Skinner serán aquellas actuaciones que propicien y justifiquen la conformación de una nueva sociedad y que a su vez se desempeñen como reforzadores de comportamiento social.

La manera de enseñar por preferencia para Skinner es la que proviene del método científico, expresado en términos de las modificaciones de la conducta posibles en un ambiente controlado, el cual podemos entender como un laboratorio. Tal como se mencionó anteriormente, un estímulo produce una respuesta y el refuerzo inmediato promueve el aprendizaje. Entre ese estímulo y la respuesta respectiva se introduce la modificación conductual y transitivamente según esta manera de enfocar el trabajo de Skinner, la didáctica.

La enseñanza programada es el método escogido por Skinner y está basada en su concepto del condicionamiento operante, (definido más adelante) lo cual debe producir una conducta programada.

Skinner crea, como se describió al inicio de este trabajo, "La Máquina de Enseñar", un recurso para la enseñanza de las materias de álgebra y gramática, en el aula de educación superior, por tanto los contenidos para esta enseñanza ya estaban predefinidos en los currículo y programas de aprendizaje correspondiente. La evaluación del aprendizaje se daba por el número de aciertos efectivos logrados por el aprendiz durante la interacción con el recurso, en caso de que la respuesta no fuese la correcta se le proporcionaba al alumno un refuerzo y una nueva 
oportunidad para su selección adecuada impidiendo avanzar a nuevas temáticas si la anterior no había sido aprobada, una prelación obligada.

Para Skinner, la relación pedagógica, es ese proceso educativo, sus actores: docente y alumnos desempeñan roles que son precisos para que el aprendizaje se produzca según el esquema Estímulo-Conducta-Refuerzo (E-C-R) en un escenario educativo:

- El maestro es responsable del reforzamiento del acierto y este refuerzo debe ser inmediato durante una contingencia. Aunque esto es complicado más que todo cuando el maestro maneja grupos grandes, Skinner considera necesarias estas condiciones: "Quizá la crítica más seria contra el actual sistema de clases es la relativa falta de frecuencia en el reforzamiento" (Bowen, ob.cit), por ello logra convenir la idea del reforzamiento en bloque de tareas.

- El papel supervisorio del maestro es importante durante la clase, sin embargo esto no es condición en el avance del aprendiz.

- Cada niño progresa a su propio paso, más no se deja de lado el carácter prescriptivo de la instrucción, una planificación no sistémica que sea responsable por el objetivo logrado.

- El docente debe sostener una homeóstasis en la clase proveyendo de complejidad o simpleza y guiando al alumno por caminos interesantes y diferentes en su instrucción. El maestro es el guía del proceso: "puede acelerar el proceso o dirigirlo ligeramente por otros lados, pero no puede enseñar sino sólo auxiliar al alumno para que él aprenda" (Bowen, ob.cit; p. 288)

- La relación del docente con el alumno es de suma importancia ya que en estas relaciones sociales surgen situaciones que no se pueden sustituir por el uso de un aparato mecánico. El auxilio instrumental optimiza la relación docente alumno pero no es la relación en sí misma.

- El docente es el controlador de la enseñanza y el responsable que el alumno "para sus conocimientos" como en la mayéutica socrática: "...mientras más enseña el maestro, menos aprende el alumno:" (Bowen, ob.Cit.; p. 287) - Pareciera en este punto que Skinner tiende a simpatizar con la idea del Insight pero aclara que "una mano" lo salvó de ello.

- El alumno es un guiado por el maestro, es responsable de un logro que testimonie su libertad y sus valores, y es exonerado de culpa cuando las "cosas salen mal".

Durante nuestros años de estudio se nos hace referencia al Conductismo, corriente que además es posterior al constructivismo, y a Skinner como uno de sus más destacados representantes, como una corriente psicológica tildada de retardataria, primitiva y hasta inadecuada tras la premisa de que su obtención 
derivó de aplicaciones a animales más que en seres humanos y que se basa en instrucciones, aplicaciones y desmereciendo el proceso revisa solos resultados. Durante la vida académica el Constructivismo se alza como bandera para todo aquel profesional que promueve una educación de avanzada y hurga en la geist de sus alumnos para que éstos de alguna manera mayéutica produzcan un nuevo conocimiento.

Lo cierto es que se siente, sin mediar muchas palabras, una satanización hacia el conductismo y a quien haga uso de su pedagogía, un retrógrado. "Somos hijos del conductismo", me decía constantemente en postgrado un profesor de Teorías del Aprendizaje, "estamos acostumbrados a aprender así", tampoco lo creo. Lo que si creo es que, dependiendo de la intención docente una manera de enseñar y de aprender se justifica siempre y cuando se promueva el proceso educativo.

Quizás para un niño en etapa inicial, aplicar la pedagogía de Skinner no sea tan adecuado como hacerlo a un trabajador que requiere aprender una rutina o secuencia de tareas. Este último no necesita interiorizar la experiencia, quizás necesite de poca experiencia previa, no necesite "darse cuenta de...", tan solo requiere memorizar pasos y en esto realizar su labor; por lo que una corriente conductual le haría ganar tiempo y aumentar la eficacia en su desempeño, entonces ċla pedagogía de Skinner, es mala?.

Si bien todos los aspectos del hecho educativo son curiosos la evaluación lo es aún más. - ¿Cuánto saqué Profesor?, es la frase que a muchos reconforta y a otros estremece pero que todos necesitan. No importa cuánto trates de proporcionar un feedback adecuado y oportuno, tampoco cuánta motivación promuevas al grupo, el número es la verdad heredada de la generación de la medición que se transversaliza de buena o mala manera en nuestra educación. Esto me lleva a pensar, ¿existirá entonces una aplicación pura de una pedagogía?, ¿será posible lograr deshacerse por completo de la propuesta de Skinner en Educación?, tal vez sea temerario decirlo pero no es algo que veo a corto plazo. Este fenómeno lo he observado en todos los niveles educativos, es trascendente y por más que se quieran introducir otras pedagogías la secuela conductual está presente en muchos detalles.

\section{LA PEDAGOGÍA INSTITUCIONAL: GEORGES LAPASSADE}

Georges Lapassade, profesor emérito de la Universidad de París, filósofo, psicosociólogo y etnólogo francés que en el mundo pedagógico se le conoce como uno de los fundadores y de los más notables animadores de la Pedagogía Institucional, la llamada Autogestión Pedagógica. Durante los años sesenta, junto con M. Lobrot, R. Lourau, R. Hess realizó experiencias autogestionarias en escuelas y otras instituciones sociales, que acompañaron siempre de una reflexión sobre los 
principios de su actuación y sobre los resultados obtenidos, hasta el punto de llegar a construir una corriente pedagógica de personalidad bien definida.

Si ubicamos el contexto histórico en el cual crece y se desarrolla el autor sería interesante ir hasta finales del S. XIX, período durante el cual el movimiento obrero planteó la gestión de sus productos por parte de sus trabajadores como reivindicación y en manifiesto de incomodidad contra el poder omnipresente del Estado, hecho éste respaldado por teóricos socialistas como Marx y Fourier, y que pueden mostrarse materializado en la Comuna de París (1871) donde según el mismo Marx, queda demostrada la capacidad de "autogobierno" de los trabajadores comunales y por ende, una muestra de sociedad autogestionada la cual puede servir de semillero que proporcionará ideas sobre las cuales cimentar las directrices de la Pedagogía Institucional. Para este momento, la revolución gestada tiene como función destruir la superstructura existente para así dejar a flote la espontaneidad creadora del cuerpo social en este caso comunal; "La autogestión es, antes que nada, esa liberación de las fuerzas instituyentes." (Lepassade, 1986)

Durante los años veinte del siglo pasado, algunos psicólogos y sociólogos proporcionaron la prueba experimental de que la autogestión que se dio y registró en la Comuna de París podía ser una condición de rendimiento alejado de las creencias del surgimiento de un desorden improductivo.

La investigación de L. Coch y J. French (1948) demostró que los cambios son aceptados y realizados con mayor facilidad cuando son decididos por los propios interesados - en este caso por los propios trabajadores - y no por la burocracia de la empresa. La autogestión pedagógica se ubica en ese contexto y en ese punto de convergencia, tal como lo describe el mismo Lapassade:

"Nuestro trabajo de investigación sobre las instituciones educativas fue preparado, también, por los progresos realizados en otro sector - el de la terapéutica institucional - en el que encontró, además, puntos de apoyo. Progresivamente, fuimos descubriendo que nuestras críticas, primero, y nuestras construcciones teóricas y metodológicas, más tarde, coincidían con el camino recorrido desde 1942 por los psiquiatras institucionalistas. Como ellos, después de haber practicado en pedagogía los métodos de grupo y las técnicas Freinet, descubrimos, pero veinte años más tarde, en 1962, que el significado final de lo que ocurre en el campo de la formación sólo surge a la superficie a partir del momento en que nos decidimos a tomar en cuenta su dimensión institucional. Los psicoterapeutas lo habían descubierto en el ejercicio de las terapias grupales, cuya eficacia se veía limitada, si no abolida, por el cuadro institucional; y nosotros, por nuestra parte, tuvimos que admitir que los grupos de formación veían limitada su eficacia por la ignorancia, en 
los mejores formadores, del análisis institucional de las estructuras que organizan la formación y en las que esta se apoya". (p. 4)

Puede hablarse entonces del nacimiento de la Pedagogía Institucional, y con ella, su instrumento técnico: la Autogestión Pedagógica, desde la influencia del pensamiento socialista en la cuna de Marx, Engels, Fourier, Proudhon conjugado con técnicas propuestas y aplicadas por Freinet y que a diferencia de Rogers el cual reduce la influencia educativa a la orientación de las relaciones interpersonales, la Pedagogía Institucional cree que son las instituciones sociales y escolares aquello que ejerce una presión educadora insoslayable. Es decir, la educación tendería a ser el resultado de las influencias que ejerce el complejo institucional en que el niño está inserto.

La Autogestión Pedagógica es considerada por Lapassade como:

"...un sistema de educación en el cual el maestro renuncia a transmitir mensajes y define, en consecuencia, su intervención educativa a partir del médium de la formación y deja que los alumnos decidan los métodos y los programas de su aprendizaje."

En su definición Lapassade emplea la terminología del médium y de mensaje por lo que añade: "...toda pedagogía consecuente busca, antes que nada, actuar sobre el medio de la formación, sobre la institución, sobre los dispositivos por los que pasan los mensajes."

Por tanto, lo que educa es la organización grupal de los educandos: subgrupos, líderes, redes comunicativas, etc.; la organización del aula: normas, horarios, métodos de trabajo, evaluaciones, etc.; la organización de la escuela: programa educativo e ideario, dirección, claustro, edificio, etc.; y, por último, la organización del sistema educativo y de la sociedad en su conjunto: legislación, planes de estudio, inspección, u otras instancias más alejadas del sistema escolar. La Pedagogía Institucional centra sus trabajos pedagógico-educativos en el análisis y las transformación de ámbito institucional y tiene como finalidad la autogestión del alumno promoviendo la libertad de la clase en todos sus componentes y actores, la responsabilidad en el cumplimiento de las tareas planteadas por la clase y la cooperación tanto es aspectos de desarrollo de la clase como en la evaluación.

La autogestión significa el desenmascaramiento de la violencia institucional, cuando por ella se entiende: a) mejorar el clima educativo y b) facilitar las relaciones interpersonales ante instituciones represivas.

En la autogestión pedagógica, los educadores y los educandos no se encuentran sometidos a reglamentos y a programas, sólo normas que ellos crearon, que se originan dentro del grupo y clase cuyo trabajo cotidiano 
determinan. Mientras que las las instituciones sociales son coercitivas y se hallan fuera del alcance del grupo las instituciones internas en la Pedagogía Institucional pueden dar lugar a una actividad instituyente de los educandos y esto define la Autogestión Pedagógica. (Lapassade, Ob.cit).

La clase en Autogestión Pedagógica se asemeja a una cooperativa en la cual se administra colectivamente el conjunto de las actividades de la clase (sus útiles: los libros, los temas, etc.). Como se mencionó en el punto anterior, en la Autogestión Pedagógica lo que educa es la organización grupal de los educandos en subgrupos, líderes, redes comunicativas, y la organización del aula como son las normas, horarios, métodos de trabajo, evaluaciones, todo convenido previamente por la clase. Por tanto cada aspecto de la educación es una oportunidad de aprendizaje.

Para Lapassade, el educador ya no enseña directamente como en otras propuestas pedagógicas. En la Autogestión el educador se desempeña en tres posibles tendencias:

1. Los educadores proponen al grupo de educandos modelos institucionales de funcionamiento en autogestión. Esta tendencia fue iniciada por Makarenko (1917) siendo de tipo autoritaria en la concepción y la institución de la autogestión.

2. Corresponde a las concepciones norteamericanas del "autogobierno" y diferentes intentos de autoformación. Según el mismo Lapassade: "...la obra fundamental de Freinet es, a mi juicio, la que mejor define esta orientación". El principal aporte que le otorga Freinet a esta tendencia es la invención de nuevos medios educativos como lo son: el texto libre, el diario, la correspondencia, así como los consejos de clase y de autogestión. Proviene del Plan Dalton que a su vez surge del Método Montessori, resumidos en dos puntos:

a. El método del Contrato

b. La enseñanza individualizada, de autogestión y de autocorreción. Complementariamente menciona Lapassade (ob. Cit):

"La pedagogía institucional del Groupe Techniques Educatives (F. Oury y A. Vásquez, M. Labat, B. Bessi y R. Fon vieille, en algunos aspectos) surgió de esta corriente. La importancia esencial del movimiento Freinet consiste en haber otorgado la mayor atención, en un primer momento, a lo que Makarenko llamaba la base material de la institución. Luego, en la invención de los nuevos medios, y finalmente, la importancia otorgada al Consejo lo que permitió la transición, tal como acabamos de destacarlo, de la autogestión restringida (la cooperativa) a la autogestión generalizada, es decir, ampliada a toda la vida de la clase."(p.7)

3. La Orientación Libertaria: es una tendencia no instituyente en la cual los educadores se abstienen completamente de proponer cualquier tipo 
o modelo insitucional y solo dejan que el grupo de los educandos encuentre e instale instituciones internas. En la clase, en autogestión, la antigua relación educador-educando ha sido abolida.

En cuanto a la escuela, ésta es una institución social regida por normas relativas a la obligación escolar, los horarios, la distribución del tiempo de trabajo, etc. En consecuencia, la intervención pedagógica de un educador (o de un grupo de educadores) sobre un conjunto de educandos se ubica siempre en un marco institucional: la clase, la escuela, el liceo, la facultad. (Lapassade, Ob.cit). Pero, en oposición a Pedagogía Tradicional de las instituciones, se propuso que se designara con el nombre de Pedagogía Institucional a una pedagogía en la cual las instituciones internas de la clase se transforman simplemente en medios cuya estructura es susceptible de ser transformada.

Para finalizar, la Autogestión Pedagógica, la cual ubico cronológicamente más próxima al pensamiento de Skinner, la concibo como la radicalización y especialización del movimiento de Escuela Nueva desde las propuestas de Freinet y Dewey. De hecho, Lepassade quien se esmera en la observación de todo el proceso pedagógico, durante la explicación de su tendencia hace referencia a Freinet en tanto este último se fija en su guía de actividades como posibles acompañantes del desarrollo inicial del alumno en el aula, siendo éste un ser activo de su propia educación y el docente un ente pasivo pero interventor en caso de que el alumno y la situación pedagógica así lo requiera.

La Pedagogía Institucional pareciera ser trascedente a la Escuela Nueva y ni se toca con el Conductismo ya que no solamente se vale de la libertad y de la experiencia tanto del alumno como del docente tal como en su momento lo pretendió Dewey, sino que también le da un valor fundamental a la institución y al medio conformador de la gnosis del alumno. Sin desmerecer las instituciones externas ya explicadas anteriormente, las instituciones internas o de la clase son las que definiran el proceso educativo del alumno. Esto hace que, a pesar de respetar las instituciones externas se consideraron antiautoritarias en la medida de que la libertad consensuada en la que se desarrolla la educación en el aula despierten la creatividad, responsabilidad, organización del tiempo y otros aspectos no menos importantes en el alumno y en el docente.

\section{LA PEDAGOGÍA CRÍTICA: PAULO FREIRE}

Freire, hijo de una familia de clase media pobre de Recife, Brasil; conoció la pobreza y el hambre, una experiencia que formaría sus preocupaciones por los pobres y que le ayudaría a construir su perspectiva educativa. Se matriculó en la 
Universidad de Recife en 1943 en la Facultad de Derecho, donde estudió Filosofía y Psicología del Lenguaje al mismo tiempo. Se incorporó en la burocracia legal pero nunca practicó la abogacía sino que prefirió dar clases de portugués en secundaria.

Trabajando principalmente entre los pobres que no sabían leer ni escribir, Freire empezó a adoptar un método no ortodoxo de lo que puede ser considerado una variación de la Teología de la Liberación, una corriente teológica la cual intenta responder a la cuestión de cómo ser cristiano en un continente oprimido y cuyo representante más reconocido, Leonardo Boff, fue su coterráneo. En esa época, leer y escribir era un requisito para votar en las elecciones presidenciales brasileñas y no hacerlo resultaba una forma de opresión ante una clase dominante que si tenía oportunidades de hacerlo.

Paulo Freire es ubicado dentro de la Pedagogía Crítica, una Pedagogía derivada de las Ciencias Críticas, un pensamiento emancipatorio en el cual un curso de acción debe ser evaluado en acción. Esta corriente Crítica va más allá de la Hermenéutica ya que además de observar la situación y comprenderla intenta cambiarla o modificarla ya que durante su indagación encuentra desventajas entre sus actores las cuales están íntimamente ligadas a desigualdades de Poder por lo que en la Pedagogía Liberadora de Freire se promueven los valores de igualdad, fraternidad y respeto. Como consecuencia de su derivación de las Ciencias Críticas su atención se centra en la importancia de las interacciones entre los individuos de las sociedad, las creencias y cognición de los actores y, por último, el uso que se le da al lenguaje en el proceso de comunicación.

La obra de Freire está íntimamente ligada por el interés de que se desarrolle en el hombre una educación liberadora por lo que está dirigida a los oprimidos, por ello fija como objetivos de su propuesta pedagógica: a) la humanización del Ser y que éste se realice en su vocación, y b) desarrollar en el educando una conciencia crítica, el sujeto que opera y transforma el mundo. (Freire y Fiori, 1980; p. 43) La crítica de Freire es hacia la educación tradicional o llamada por él mismo una concepción bancaria de la Educación que predominada en Brasil para el momento que escribe sus obras. En esta obra Educación Liberadora, Freire se ocupa de la alfabetización de adultos. Su interés se muestra desde la premisa de que el pueblo es el motor de los cambios históricos pero ¿cómo los oprimidos podían ser actores de un proceso de cambio si más de la mitad son analfabetas?, por lo que la alfatización se convirtió en un reto pues "el analfabeta era considerado un enfermo con una enfermedad que debe ser curada". (Freire y Fiori, ob.cit.; p. 53)

Para Freire la didáctica se fija en el diálogo, por ello se promueve una Educación Dialógica frente a una Educación Bancaria trabajando a partir del capital cultural del educando. Las actividades educativas son de diálogo con el alumno para así incorporar el bagaje cultural del alumno al currículo y evitar incluir 
significados impuestos por alguna cultura dominatoria que pretenda ser más poderosa que la cultura proveniente del contexto del educando.

En las actividades, Freire persigue a través de estrategias y actividades problematizadoras la estimulación de la creatividad, la búsqueda, el riesgo a crear y la admiración por el mundo: "Esta concepción humanista, que rechaza los depósitos, la mera disertación o narración de los trozos aislados de la realidad, se realiza a través de la constante problematización del hombre mundo." (Freire y Fiori, ob.cit; p. 49)

Freire no especifica sobre la evaluación del proceso educativo, sin embargo, podemos inferir que la revisión de dicho proceso o su valoración sucede en cada momento de la educación puesto que, como lo diremos más adelante, toma la idea de Freinet de la dialéctica teoría-práctica lo que introduce un carácter sistémico a la educación. Así mismo, es posible deducir que la efectividad o eficacia del proceso educativo se mostrará en la medida que el alumno sea un factor de cambio en su contexto, reconozca las variaciones de la dominación y promueva cambios para su liberación y así poder ser evaluado.

En cuanto al contenido de la enseñanza, éste sale del contexto del individuo lo que se puede entender como el desarrollo del currículo oculto. En esta obra, que como dijimos trata la alfabetización de adultos, Freire se plantea enseñar la lectura y escritura y lo hace a través de frases que tienen un significado para los educandos lo que un tema fijado toma su contenido del medio de desarrollo de sus participantes de una manera específica, no generalizada. Al este respecto Freire describe:

"Y aun cuando las palabras del silabario, los textos con ella elaborados -lo que raras veces ocurre- coincidan con la realidad existencial de los alfabetizandos, son palabras y textos regalados, como clisés, y no creados por aquellos que deberían hacerlo. En general, sin embargo, tanto las palabras como los textos de los silabarios nada tienen que ver con la experiencia existencial de los alfabetizados. Y cuando lo tienen, se agota esta relación al ser expresada en forma altamente paternalista, de lo que resulta tratar del adulto de manera que no osamos siquiera llamar infantil" (Freire y Fiori, ob.cit; p. 55)

Para Freire la relación pedagógica que se da en el contexto educativo es bastante diferente a lo cómo se entendían estos roles alumno docente en la educación tradicional. Las posiciones de los actores educativos es cónsona con la corriente de pensamiento crítico que se desarrollan en cada obra de educación liberadora y, desde la concepción humanista resulta en la relación simbiótica de un educador-educando con un educando-educador, que puede resumirse en: 
1. Nadie educa a nadie

2. Nadie se educa solo

3. Los hombres se educan entre sí, mediatizados por el mundo. (Freire y Fiori, ob.cit; p.50)

Es importante destacar que en la Pedagogía de Freire el educador es crítico de sí mismo y de su práctica constante y es reflexivo; a partir de estas características se desarrolla en su vocación reivindicando su rol docente y la autonomía de la escuela.

El ambiente de aprendizaje es el mismo mundo y las relaciones que en él surgen, en lo que Freire se diferencia con la pedagogía de Neill por ejemplo. Sin embargo, de Freinet retoma la dialéctica teoría-prácticacomo parte de su experiencia reflexiva, y a su vez la experiencia reflexiva pareciera retomada de Dewey.

Paulo Freire es de los pedagogos más cercanos a esta época y lo considero un poco más trabajado cuando cursamos estudios académicos en Educación que Freinet y Neill, por ejemplo. Sin embargo, y como lo he mencionado en otras oportunidades, el estudio de estas corrientes durante la educación formal se hace a vuelo rasante sin develar el pensamiento del pedagogo, solo conformados con las principales características de su propuesta a manera de receta de cocina.

En esta oportunidad considero sumamente interesante el manejo que hace Freire del oprimido en el contexto educativo, proponiendo que ante una resistencia surge una liberación a la dominación en la que está un alumno sometido por el opresor docente-contenido pero, ¿cómo puede darse esta liberación si el oprimido no es formado para tal fin? Esta es la pregunta problematizadora que sirvió de disparador para presentar su propuesta en la obra de Educación Liberadora. Este breve análisis trajo a mi pensamiento algunas ideas de Michel Foucault sobre los procesos de dominación - represión presentado en términos de lucha - sumisión.

El docente, y en algunos casos el contenido, es visto como una forma de dominación la cual intenta apoderarse del alumno y este así lo permite. Por ello, el rol del docente para el autor cambia notablemente y pasa a ser un ser reflexivo y, en esa dialéctica de reflexión - acción - reflexión mejora su práctica docente. En algunos momentos de la lectura muestra al educador como un subversivo, como un individuo que va a ir en contra de posturas preestablecidas y autoritarias, intentando en su causa la abolición de los desequilibrios de poder y la liberación de la alienación que se produce en la sociedad, por ende, Freire pretende una declarada mejora social, un socialismo. Esta línea de pensamiento guarda continuidad con los escritos de Michael Apple en ciertas revisiones que hice sobre las políticas del texto escolar. 
En cuanto a la Concepción Bancaria de la Educación, queda claro que la crítica de Freire va directamente contra esta posición hasta cómoda tanto de los educadores como de los alumnos. A este respecto, Freire hace mención de un docente poseedor del conocimiento y un alumno pasivo, un simple receptor del conocimiento que posee el docente y subyugado a su criterio, en el sentido de que el alumno solo aprenderá lo que el docente quiere siendo esto entonces una manifestación expresa de dominación. Esta corriente llamó mucho mi atención. A través de la observación de las clases de docentes de bachillerato en mi contexto de trabajo de hace años, se deja ver que la concepción bancaria de la educación es inminente e instaurada en programas educativos, currículos, instituciones educativas...

Normalmente no se le da oportunidad al alumno de la crítica, quizás por temor por parte del docente o por mal entendimiento de lo que una crítica representa, pero tampoco se le ha formado para fijar posición frente a situaciones sociales que manifiesten dominación o desigualdad de oportunidades. En este punto, el alumno está hasta cómodo siendo esa alcancía de conocimiento, descansa sobre una zona de confort en la cual obtiene los contenidos ya trabajados y elaborados, entonces, ¿cómo salir de una dominación si no hay postura crítica en la Educación ni por parte de maestros ni de alumnos?

Me ocupé también de conversar con docentes de educación primaria a manera de indagar sobre el conocimiento que tenían de Paulo Freire. En su mayoría reconocieron la importancia de Freire en su práctica pedagógica, pero cuando se profundiza se llega solo a técnicas para enseñar a leer, nada más allá, y surgen más preguntas: ¿tristeza o comodidad?... ¿hay diferenciación entre pedagogía, didáctica y educación? No lo creo; tengo ahora más preguntas que cuando inicié la lectura y análisis de las obras.

\section{BIBLIOGRAFÍA}

Philosophy and Democracy. J. A. Boydston (Ed.) The middle work, 1899-1924, Vol. 11, 1918-1919. Carbondale, Southern, Illinois University Press.

Bowen, J. (1986) Teorías de la Educación: innovaciones importantes en el pensamiento educativo occidental. Mexico:Limusa

Dewey, J. (1938) Experiencia y Educación. Buenos Aires:Losada Freire, P. y Fiori, H. (1980) Educación Liberadora. 5ta. ed. Bilbao:Zero

From absolutism to experimentalism. J. A. Boydston (Ed.) The later work, 19251953, Vol. 5, 1929-1930. Carbondale, Southern, Illinois University Press.

L. Coch y J. French jr., (1948) Overcoming resistance to change. Vol. 1, pp. 512532 
Lapassade, G. (1986). Autogestión Pedagógica: Un sistema en el cual los educandos deciden en qué consiste su formación y la dirigen. Barcelona:Gedisa.

Loeb, J. (1900) Einleitung in die vergleichende Gehirnphysiologie vergleichende und Psychologie, Leipzig: JA Barth, 1899 - Inglés ed, fisiología comparada del cerebro y la psicología comparada, New York: Putnam.

Pavlov, I. P. (1927) Conditioned reflexes: an investigation of the physiological activity of the cerebral cortex. Edited by Anrep GV. New York, Boyer.

Rockefeller, S. (1991) John Dewey. Religius faith and democratic humanism. New York:Columbia University Press.

Watson, J. B. (1928). Psychological care of infant and child.

\section{Pedro Andrés Certad Villarroel}

Licenciado en Educación con Postgrado en Tecnología, Aprendizaje y Conocimiento - Graduado con Honores, egresado de la Universidad Metropolitana. Aspirante a Doctor en Educación en la Universidad Central de Venezuela. Profesor de Química Inorgánica y Orgánica. Profesor agregado y Coordinador de Educación y Tecnología adscrito al Departamento de Ciencias de la Educación de la Universidad Metropolitana en Caracas, Venezuela. Miembro del equipo investigador de la Célula Universitaria Iberoamericana de Investigaciones Sociológicas "Concilium". Evaluador-Revisor en el sistema de arbitraje por pares ciegos de las revistas Vivat Academia (ISSN 1575-2844) y Revista de Comunicación de la Sociedad Española de la Comunicación Iberoamericana (ISSN 1576-3420). Miembro del Comité Organizador y del Comité Científico para el Congreso Universitario Internacional sobre Contenidos, Investigación, Innovación y Docencia - CUICIID 2011- de la Facultad de Ciencias de la Información de la Universidad Complutense de Madrid. 